\title{
A new approach to complete graph
}

\author{
Basheer Ahamed Mohideen \\ Department of Mathematics, Faculty of Science, University of Tabuk, Tabuk, Kingdom of Saudi Arabia
}

Received: 31 August 2016, Accepted: 17 December 2016

Published online: 26 February 2017.

\begin{abstract}
A graph consists of a nonempty vertex set and an edge set, denoted $\mathrm{G}=(\mathrm{V}, \mathrm{E})$. An unordered pair of edge v,w is known as an undirected edge and an ordered pair of edge $(\mathrm{v}, \mathrm{w})$ is known as a directed edge. A graph with directed edges is called a directed graph, simply a digraph; otherwise an undirected graph. Usually, an undirected graph is simply known as a graph. This paper gives a new approach to complete graph and investigates some results related to this idea.
\end{abstract}

Keywords: Graph, complete graph, regular graph, loop-full complete graph.

\section{Introduction}

The theory of graphs was established in $18^{\text {th }}$ century by Leonhard Euler on the Königsberg Seven Bridges problem. A graph is an expedient way of representing information involving relationship between objects. The objects are represented by vertices and relations by edges. Graphs can be used to model many types of relations and processes in physical, biological, social and information systems. Many practical problems can be represented by graphs. In computer science, graphs are used to represent networks of communication, data organization, computational devices, the flow of computation, etc. A graph structure can be extended by assigning a weight to each edge of the graph. Graphs with weights (or weighted graphs) are used to represent structures in which pair wise connections have some numerical values. For example, if a graph represents a road network, the weights could represent the length of each road.

Most of the articles in graph theory are considered only simple graphs. A simple graph is a graph that has no loop(s) and/or no parallel edge(s). When we construct a network of main cities connectivity in a country, we just think a city as a crossing point (vertex). Recent days, every metropolitan city consists of many malls and historical places to visit by a tourist. Therefore a tour operator wants to make sure a tourist to visit all historical places and shopping malls in a big city with maximum gain and minimum expenditure, in this situation the tour operator can make a circle (loop) to cover maximum places in that city, so we need to consider a self-loop for each vertex (point) and include this concept in complete graph. In this paper, we consider $G=(V, E)$ is a finite undirected connected graph without multiple edge(s).

\section{Preliminaries}

In this section, let us recall some basic notions and their related results in graph theory, which are referred in [1-5].

A graph is an ordered pair $G=(V, E)$, where $V$ is the nonempty set of vertices of $G$ and $E \subseteq V \times V$ is the set of edges of $G$. Two vertices $v$ and $w$ in an undirected graph Gare said to be adjacent or neighbors in $G$ if $\{v, w\}$ is an edge of $G$. The 
concept of incidence associates an edge to the nodes that are connected by that edge. For example an edge $e=\{v, w\}$ is incident to the nodes $v$ and $w$. The order of $G$ is number of vertices in $G$, denoted $|V|$. The size of a graph is $|E|$, its number of edges. The end vertices are vertices connected by an edge. An edge has same end vertex is called a self-loop (simply loop), degree of a loop is counted two. Two or more edges are having same end vertices are called parallel edges (or multiple lines). A simple graph is a graph that has no loop(s) and/or no parallel edge(s).

A complete graph is a graph in which each pair of vertices is joined by an edge, a complete graph of $\mathrm{n}$ vertices is denoted by $K_{n}$, it has $\left(\begin{array}{l}n \\ 2\end{array}\right)$ edges. Note that a complete graph of odd vertices is odd complete graph; similarly a complete graph of even vertices is even complete graph. The neighborhood of a vertex $v$ in a graph $G$ is the induced subgraph of $G$ consisting of all vertices adjacent to $v$ and all edges connecting two such vertices. The neighborhood is often denoted $N(v)$. The degree (or valency), denoted $\operatorname{deg}(v)$ of vertex $v(\operatorname{simply} d(v)$ ) is the number of edges incident on vor equivalently, $\operatorname{deg}(v)=|N(v)|$. A basic result consists of degree and edge cardinality is $\sum_{v \in V} d(v)=2|E|$. A vertex of odd degree is called an odd vertex. A vertex of even degree is called an even vertex. The set of neighbors, called a (open) neighborhood $N(v)$ for a vertex vin a graph $G$, consists of all vertices adjacent to $v$ but not including $v$, that is, $N(v)=\{w \in V: v w \in E\}$. Note that, For an edge $\{v, w\}$, use simply $v w$. When $v$ is also included, it is called a closed neighborhood, denoted $N[v]$, that is, $N[v]=N(v) \cup\{v\}$. A regular graph is a graph where each vertex has the same number of neighbors, that is, all the vertices have the same open neighborhood degree. A $k$-regular graph is a graph in which each vertex has degree $k$.

A walk is an alternating sequence of vertices and edges in a graph. A trail is a walk that does not contain repeated edge(s). An Euler trail is a trail in a graph that visits every edge exactly once. Similarly, an Euler circuit is an Euler trail that starts and ends at the same vertex. An Euler graph is a graph that contains an Euler circuit. A connected graph is Euler graph if and only if every vertex has even degree. An adjacency matrix is a square matrix used to represent a finite graph. The elements of the matrix indicate whether pairs of vertices are adjacent or not in the graph. A unit matrix is a matrix consisting of all 1's, denoted $J_{m \times n}$, where $m$ is number of rows and $n$ is number of columns. A unit square matrix of order $n$ is denoted by $J_{n}$.

\section{Loop-full complete graph}

This section firstly introduces odd and even regular graphs and secondly a loop-full complete graph. Finally, it investigates some of their properties.

Definition 1. A k-regular graph is called an odd regular if $k$ is odd number; similarly a k-regular graph is called even regular if $k$ is even number.

Example 1. $K_{3}$ is even regular, each vertex has degree two; $K_{4}$ is odd regular, because each vertex has degree 3 . In general $K_{n}$ is odd regular if $n$ is even; $K_{n}$ is even regular if $n$ is odd.

Definition 2. A graph $G=(V, E)$ is said to be loop-full complete graph (LFCG) if it is complete graph and each vertex has a loop (self-loop), a loop-full complete graph of $n$ vertices is denoted by $K(n$.

Example 2. A loop-full complete graph of 4 vertices and 10 edges (4 loops and 6 other edges), denoted as $K_{(4)}$. 


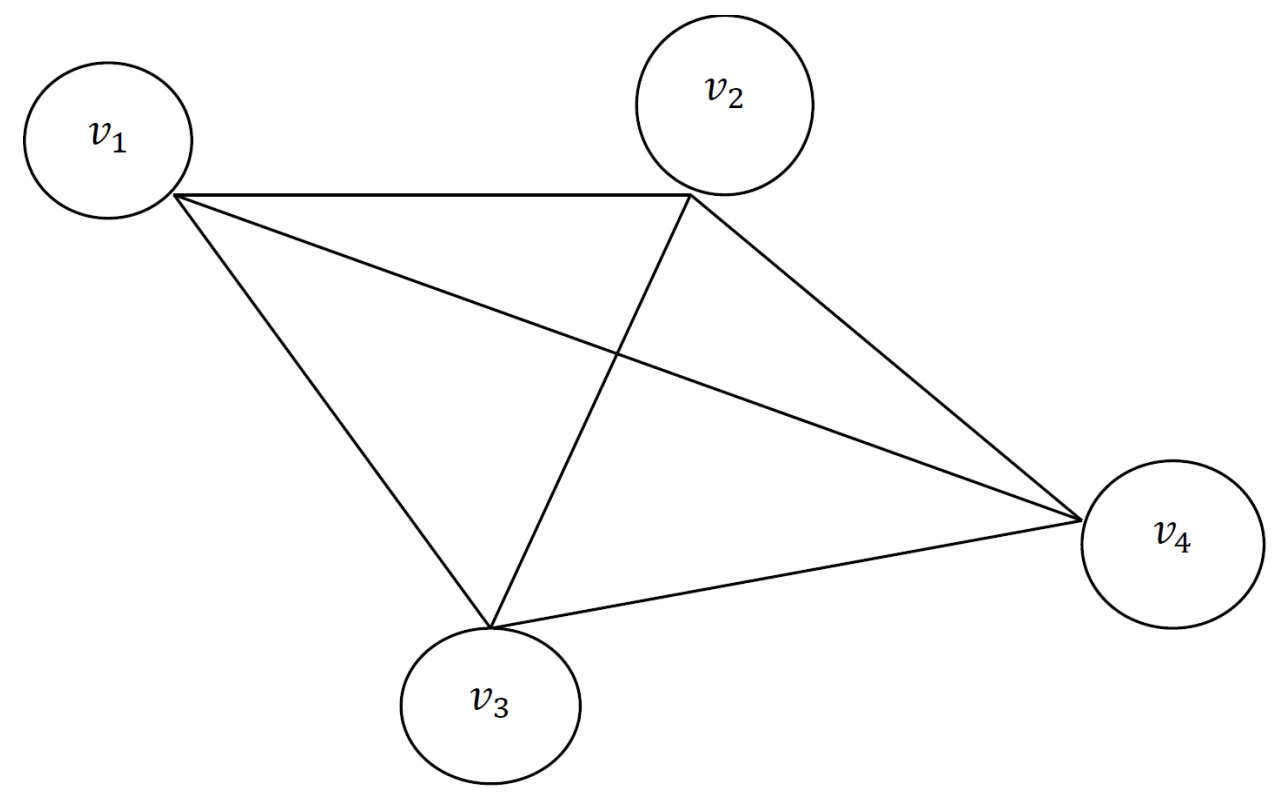

Fig. 1

Definition 3. An LFCG is said to be an odd LFCG if all are odd vertices; similarly an LFCG is an even LFCG if all are even vertices.

Example 3. $K_{(17}$ is an even LFCG; $K_{(8)}$ is an odd LFCG.

Proposition 1. In a loop-full complete graph $K \cong$, there are exactly $\frac{n(n+1)}{2}$ edges.

Proof. Let $G=(V, E)$ be a loop-full complete graph (LFCG) with $n$ vertices. Since G is complete with $n$ vertices, we have $\left(\begin{array}{l}n \\ 2\end{array}\right)$ edges. Here, $G=K(n$. Therefore, the total number of edges in $K(\mathbb{n})$ is counted as the sum of the number of edges in $K_{n}$ and $n$ edges (loops). Therefore, $G$ has $\left(\begin{array}{l}n \\ 2\end{array}\right)+n$ edges. Thus $G=K(n)$ has exactly $\frac{n(n+1)}{2}$ edges.

Proposition 2. Every loop-full complete graph $K(\mathbb{n})$ is $(n+1)$-regular graph.

Proof. Let $G=(V, E)$ be a LFCG with n vertices, that is $G=K($ n). We know that, a complete graph of $n$ vertices is $(n-1)$ - regular. For each vertex in $K \cong$, there are $(n-1)$ adjacent edges and a loop, so we count degree two for each loop and $(n-1)$ degree for its adjacent edges. Therefore, the degree of each vertex is $(n-1)+2=n+1$. Thus $K(\mathbb{n})$ is $(n+1)-$ regular graph.

Proposition 3. The adjacency matrix of a loop-full complete graph $K(\mathbb{n})$ is $J_{n}$, (i,e.,) $A\left(K(\mathbb{n})=J_{n}\right.$.

Proof. By the definition, the adjacency matrix of a complete graph of $n$ vertices $K_{n}$, we have

$$
A\left(G=K_{n}\right)=\left[\begin{array}{cccc}
0 & 1 & \ldots & 1 \\
\vdots & \vdots & \ddots & \vdots \\
1 & 1 & \ldots & 0
\end{array}\right]_{n}
$$


In LFCG, each vertex has a loop, so every vertex is connected itself, we have

$$
A\left(G=K(\mathfrak{n})=\left[\begin{array}{cccc}
1 & 1 & \ldots & 1 \\
\vdots & \vdots & \ddots & \vdots \\
1 & 1 & \ldots & 1
\end{array}\right]_{n}=J_{n}\right.
$$

a unit matrix of order $n$.

Proposition 4. Every Odd LFCG has even degrees.

Proof. Let $G=K(n)$ with $n=2 k-1, k \in Z^{+}$. Then the degree of each veretex is $(n-1)+2=(2 k-1-1)+2=2 k$, which is even. Thus we get the result.

Corollary 1. Every even LFCG has even degrees.

Proof. Proof is trivial.

Proposition 5. Every Odd complete graph is an Euler graph.

Proof. let $G=K_{n}$ where $n=2 k-1, k \in Z^{+}$. Then the degree of each vertex in $K_{n}$ is even. By the result, a connected graph is Euler if and only if every vertex has even degree. Thus an odd $K_{n}$ is always an Euler graph.

Proposition 6. Every odd LFCG is Euler graph.

Proof. Let $G=K\left(\mathrm{n}\right.$ ) where $n=2 k-1, k \in Z^{+}$. Then the degree of each vertex along with self-loop is even. By the result, a connected graph is Euler if and only if every vertex has even degree. Thus an odd $K \cong$ is always an Euler graph.

\section{Conclusion}

This paper has initiated a new approach to complete graph and discussed various results using this new concept. Further, we can extend this idea to other graph theory topics as well as fuzzy graphs in near future.

\section{Competing interests}

The authors declare that they have no competing interests.

\section{Authors' contributions}

All authors have contributed to all parts of the article. All authors read and approved the final manuscript.

\section{Acknowledgements}

The author would like to express his gratitude to the Editor-in-Chief and referees for their valuable and constructive suggestions. 


\section{References}

[1] Berge, C., Theory of Graphs and its Applications, Methuen, London, 1962.

[2] Bondy, J.A. and Murty, U.S.R. Graph Theory with Applications, North-Holland, Elsevier, 1981.

[3] Chartrand, G., and Lesniak, L., Graphs And Digraphs, Chapman \& Hall, $3^{\text {rd }}$ Edn., 1996.

[4] Diestel, R., Graph Theory, Springer-Verlag, New York, 1997.

[5] Harary, F., Graph Theory, Third ed., Addison-Wesley, Reading, MA, 1972. 